\title{
Establecimiento de prioridades de investigación en la enfermedad renal crónica de causas no tradicionales en Centroamérica
}

\author{
Ludovic Reveiz', Carlos Pinzón-Flórez², Demián Glujovsky³, Vanessa Elias ${ }^{1}$ y \\ Pedro Ordunez ${ }^{1}$
}

Forma de citar

Reveiz L, Pinzón-Flórez C, Glujovsky D, Elias V, Ordunez P. Establecimiento de prioridades de investigación en la enfermedad renal crónica de causas no tradicionales en Centroamérica. Rev Panam Salud Publica. 2018;42:13. https://doi.org/10.26633/RPSP.2018.13

RESUMEN Introducción. En 2013, los Estados Miembros de la OPS reconocieron la epidemia de enfermedad renal crónica de causas no tradicionales (ERCnT) como un grave problema de salud pública. Este artículo describe el establecimiento de prioridades de investigación para abordar de manera integral la ERCnT en Centroamérica.

Métodos. Se estructuró una encuesta virtual utilizando la metodología Delphi mediante una búsqueda de estudios de investigación efectuados en Centroamérica y de agendas de investigación previas sobre la ERC. Los encuestados se identificaron en diversas fuentes. La primera ronda buscó refinar y añadir tópicos de investigación y priorizar los más relevantes. La segunda ronda priorizó los tópicos más relevantes. Se realizó un análisis por fuzzy sets para estimar umbrales de decisión y puntajes por tópico.

Resultados. La encuesta se envió a 83 personas de habla hispana y 38 de habla inglesa y respondió 46,2\%. Para la segunda ronda, se envió la encuesta a 56 personas en español y 16 en inglés que habían contestado a la la primera. Se priorizaron 18 tópicos de investigación enmarcados en 10 áreas: políticas públicas, determinantes, etiología, diagnóstico y tratamiento de la ERC, prevención primaria, prestación de servicios, recursos humanos, sistemas de información y financiamiento. Se comprobó que la investigación en ERCnT es escasa y está restringida a ciertos tópicos.

Conclusiones. Además de los factores etiológicos, se dio gran relevancia a aspectos relacionados con la respuesta de los sistemas de salud, incluidos el abordaje de la prestación de servicios, los recursos humanos, el financiamiento y aspectos ocupacionales y ambientales.

Palabras clave Insuficiencia renal crónica; investigación; prioridades en salud; sistemas de salud; servicios de salud del trabajador.

En las tres últimas décadas se ha notificado un creciente número de casos de personas que sufren un tipo específico

\footnotetext{
Organización Panamericana de la Salud,

Washington, DC, Estados Unidos de América. La

correspondencia se debe dirigir a Ludovic Reveiz.

Correo electrónico: reveizl@paho.org
}

de enfermedad renal crónica (ERC) y fallecen por esa causa en Centroamérica (1, 2). Aunque la mayoría de los países de

\footnotetext{
Grupo de Investigación en Salud, Facultad de Medicina, Universidad de La Sabana, Chía, Colombia Instituto de Efectividad Clínica y Sanitaria, Buenos Aires, Argentina.
}

Centroamérica no cuentan con sistemas de vigilancia que detecten la ERC, la tasa de mortalidad estandarizada por edad (codificada como CKD-N18) es notablemente mayor en hombres y mujeres en Nicaragua y El Salvador que en otros países de la Región de las Américas que

Este es un artículo de acceso abierto distribuido bajo los términos de la licencia Creative Commons Attribution-NonCommercial-NoDerivs 3.0 IGO, que permite su uso, distribución y reproducción en cualquier medio, siempie que el trabajo original se cite de la manera adecuada. No se permiten modificaciones a los artículos ni su uso comercial. Al reproducir un artículo no debe haber ningún indicio de que la OPS o el artículo avalan a una organización o un producto específico. El uso del logo de la OPS no está permitido. Esta leyenda debe conservarse, junto con la URL original del artículo. 
tienen un patrón de exceso de mortalidad en adultos jóvenes (2). A nivel global, la diabetes y la hipertensión son las dos causas más comúnmente reconocidas de ERC. Sin embargo, en El Salvador, Nicaragua y en otros países de Centroamérica este significativo incremento no se asocia con diabetes ni hipertensión y afecta sobre todo a hombres jóvenes trabajadores agrícolas y también a mujeres que no trabajan en el campo, pero que viven en estas comunidades agrícolas (3-6). En la Encuesta Nacional de Enfermedades Crónicas no Transmisibles en población adulta de El Salvador (20142015) se estimaron prevalencias de ERC no tradicional (ERCnT) de 7,1\% y 5,2\% en la región Paracentral y en la región Oriental, respectivamente, que contrastan con la de 2,0\% de la región Central (3).

Para identificar esta entidad nosológica se han usado diversas denominaciones: enfermedad renal crónica en comunidades agrícolas de Centroamérica, nefritis intersticial crónica en comunidades agrícolas, nefropatía mesoamericana y enfermedad renal crónica no relacionada con causas tradicionales, (7) que es como finalmente se llama en este artículo. La Organización Panamericana de la Salud (OPS), junto con otras instituciones y actores, ha elaborado definiciones de caso para fines de vigilancia de la ERCnT (8-11).

Según estimaciones realizadas por la OPS, la insuficiencia renal, específicamente la codificada bajo N18, CIE-10 y proxi para el análisis de mortalidad por este tipo de ERC, en menores de 60 años de edad es responsable de miles de muertes durante la última década en Centroamérica y revela un exceso de mortalidad en adultos jóvenes, mayor en los hombres, pero también notable en las mujeres en los países más afectados (figura 1) (12).

Las causas de la epidemia ERCnT no están del todo claras, aunque hay consenso sobre su carácter multifactorial y su relación con los determinantes sociales, ambientales y económicos de la salud. Se han postulado diferentes factores, pero los que se han reiterado con más frecuencia son la exposición a los agroquímicos y el estrés por calor-deshidratación recurrente, derivado de las duras condiciones de trabajo y las altas temperaturas a la que están expuestos los trabajadores del campo $(7,8,13-15)$. Además, las poblaciones rurales agrícolas de Centroamérica que viven en condiciones de pobreza,
FIGURA 1. Diagrama de flujo utilizado para cribar y seleccionar los artículos científicos y documentos técnicos

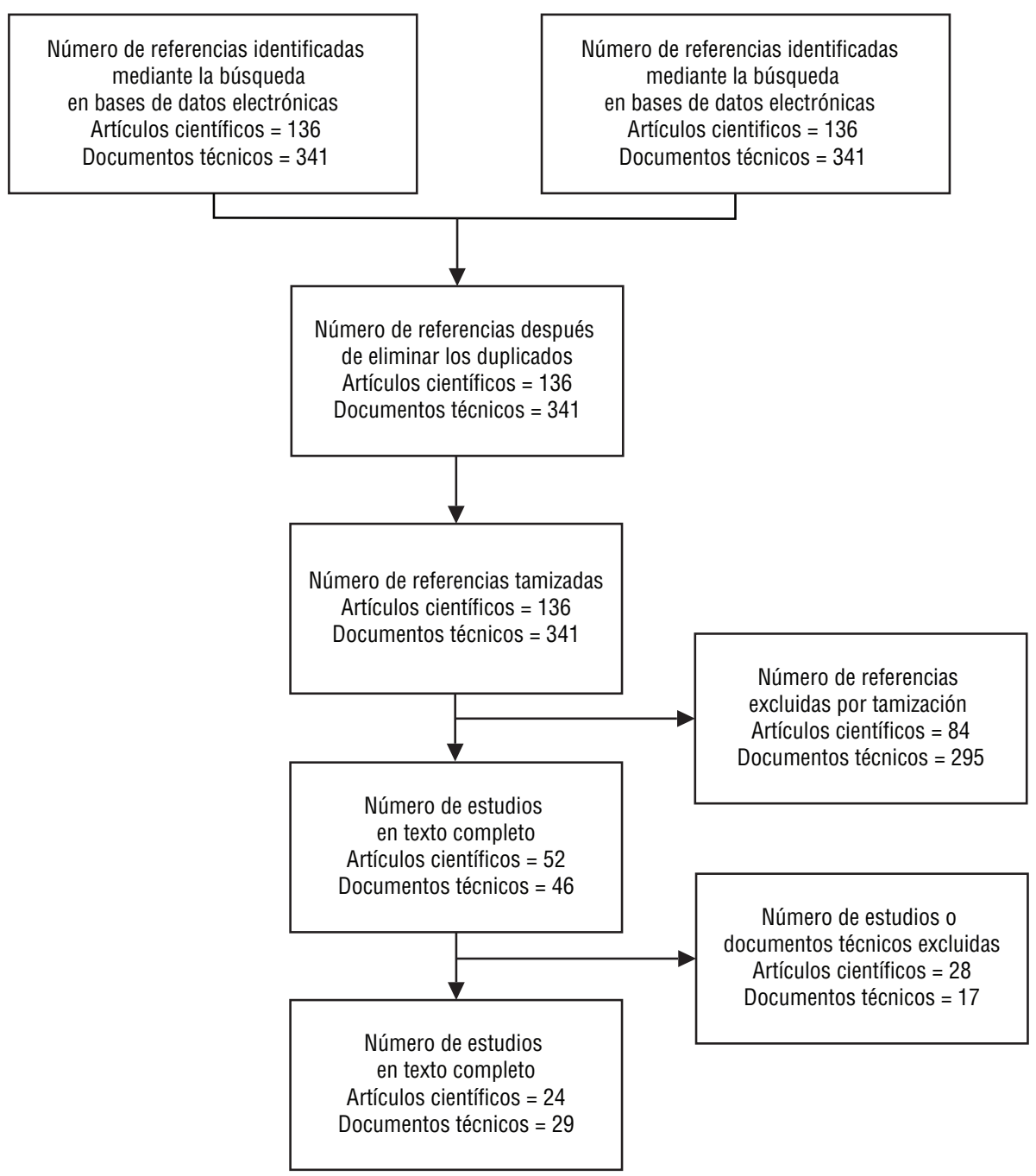

tienen un acceso limitado a los servicios de salud por las distancias entre la vivienda o el trabajo y los servicios, el limitado número de servicios con capacidad para hacer el diagnóstico y administrar el tratamiento, y los costos asociados con la búsqueda de atención. Por estas razones, estas mismas poblaciones, frecuentemente migrantes, están en situación de mayor vulnerabilidad frente a las complicaciones provocadas por la enfermedad renal $(13,15,16,17)$.

En 2013, los Estados Miembros de la OPS reconocieron la epidemia de ERCnT como un grave problema de salud pública y aprobaron la resolución CD52.R1 (18), que señala la necesidad de emprender una acción integral y coordinada. Los países se comprometieron a realizar más esfuerzos para abordar los factores ambientales y ocupacionales asociados con la enfermedad y a continuar con las investigaciones sobre sus causas y la atención médica de los pacientes afectados. Asimismo, acordaron fortalecer la vigilancia de esta enfermedad, la atención médica de los pacientes afectados, así como promover alianzas con diferentes sectores, en especial con las comunidades afectadas, para abordar este problema y mitigar con urgencia las consecuencias sanitarias, sociales y económicas de esta enfermedad (18).

A pesar del incremento en el número de investigaciones relacionadas con la ERCnT de los últimos años, existen importantes brechas de conocimiento relacionadas no solo con sus aspectos clínicos y etiológicos, sino con el abordaje desde las intervenciones de salud pública, los determinantes sociales, la prevención, diagnóstico y tratamiento de la 
enfermedad renal crónica en estas poblaciones, la prestación de los servicios de salud, y el fortalecimiento de los recursos humanos entre otros (17-25).

El establecimiento de las prioridades de investigación busca facilitar la conducción y gestión de los programas de investigación promoviendo la eficiencia y la articulación de recursos y actores regionales y nacionales, intra e intersectoriales, e interdisciplinarios, así como incorporar la evidencia científica en la práctica, la salud pública y las políticas en salud. Busca, además, facilitar el fortalecimiento de la gobernanza de la investigación, de una orientación común de centros, institutos y grupos de investigación, y de los recursos humanos en salud con las competencias adecuadas para dar respuestas a las necesidades de este problema de salud pública. El objetivo de la consulta que se presenta en este artículo fue establecer las prioridades de investigación que contribuyan a abordar la ERCnT de manera sistémica e integral.

\section{MATERIALES Y MÉTODOS}

Esta consulta se llevó a cabo a través de un proceso de varias etapas, que se describen a continuación.

\section{Búsqueda sistemática de la bibliografía}

Para identificar estudios relevantes que sirvieran de base para establecer las prioridades de investigación, se realizó una búsqueda sistemática en PubMed, EMBASE, CENTRAL, Cochrane Library, Lilacs, así como con buscadores como Google Scholar y en páginas relacionadas con el tema propuesto (desde 1 enero de 2010 hasta el 15 de julio de 2015). Se identificaron palabras clave y sinónimos utilizando el buscador PubMed. Además, se revisaron las páginas web de los ministerios de salud e instituciones pertinentes de cada uno de los países de Centroamérica y las referencias de los estudios identificados para tamizar citas adicionales. Un revisor evaluó los títulos y resúmenes identificados por la búsqueda y dos revisores evaluaron de manera independiente los estudios que cumplieron los criterios de inclusión.

Los criterios de inclusión propuestos fueron los siguientes: a) estudios que abordaron la ERCnT en países de Centroamérica y la República Dominicana, b) estudios que evaluaron o propusieron una agenda de investigación o prioridades de investigación para el abordaje de la insuficiencia renal crónica, y c) agendas de prioridades nacionales de investigación en los países involucrados. La búsqueda se limitó a informes publicados en los últimos 10 años en español, inglés y portugués.

\section{Selección de los participantes}

Se identificaron los autores de los estudios sobre ERCnT encontrados en la búsqueda sistemática de la bibliografía y se extrajeron datos sobre el país, la afiliación (para saber si tenían responsabilidades en la toma de decisiones, investigación, etc.) y la dirección de correo electrónico. Asimismo, se buscaron otros participantes potenciales a través de expertos de la OPS y de los centros de investigación en los países (Costa Rica, El Salvador, Guatemala, Honduras, México, Panamá, República Dominicana).

\section{Elaboración del cuestionario}

El grupo de autores compuesto por epidemiólogos, especialistas en salud pública, sistemas de salud, salud global y decisores en salud construyeron un instrumento tipo encuesta con el cual se trataron los diferentes temas y líneas de investigación identificadas en la búsqueda sistemática de la bibliografía. Las preguntas se redactaron y revisaron hasta que se alcanzó el consenso de todos los miembros del grupo.

\section{Encuesta a expertos}

Se llevó a cabo una encuesta estructurada usando el programa SurveyMonkey® (en idioma español e inglés) en dos rondas. La primera ronda tuvo como objetivos fundamentales refinar y añadir preguntas e identificar las áreas y los tópicos que los investigadores consideraron como relevantes. Cada pregunta se calificó a través de una escala tipo Likert, según el investigador la considerara como "muy relevantes", "relevantes", "poco relevantes", "no relevantes" o "no puedo contestar". Los participantes juzgaron la relevancia según dos criterios (26). Primero, la magnitud e impacto, es decir, que la respuesta a la pregunta contribuirá al bienestar social y tendrá potencial para facilitar el objetivo de la cobertura universal en salud de las poblaciones, además de reducir la mortalidad o la morbilidad en su país o región. Y, segundo, factibilidad, entendida como que la pregunta puede responderse con el desarrollo de investigaciones de manera ética, equitativa y sostenible dentro de contextos tecnológicos, culturales, jurídicos, políticos y socioeconómicos adecuados, y con los recursos disponibles.

El objetivo de la segunda ronda fue jerarquizar los tópicos que los investigadores consideraron relevantes siguiendo el mismo método de la primera ronda. Esta ronda se estructuró a partir de las áreas y los tópicos calificados "muy relevantes" y "relevantes" en una proporción mayor a $75 \%$ de las respuestas de todos los participantes de la primera ronda. También se incorporaron tópicos adicionales sugeridos por los participantes de la primera ronda.

Se realizó un análisis descriptivo y analítico de los datos recuperados de las encuestas utilizando el software STATA versión 12.1. La jerarquización de las prioridades se realizó utilizando el método de calificación por terciles de la tendencia de respuesta de los participantes en la categoría "muy relevantes" para cada sección propuesta. Se realizó un análisis por fuzzy sets para estimar los umbrales de decisión y las puntuaciones por cada ítem según el comportamiento de respuesta de todos los participantes (estimador k) $(27,28)$. Se conformaron las matrices decisión, para estimar el umbral (estimador k). Para el valor de cada ítem se supuso una distribución triangular de los datos. El estudio fue aprobado por el Comité de Ética de investigación de la OPS (PAHOERC).

\section{RESULTADOS}

\section{Búsqueda sistemática de la bibliografía}

Con la búsqueda bibliográfica se identificaron 136 artículos científicos relacionados con la investigación sobre la ERCnT en Centroamérica. De estos, se excluyeron 84 documentos que no cumplieron los criterios de inclusión. De los 52 artículos restantes, se incluyeron finalmente en el estudio para su evaluación 24 (figura 1). Asimismo, se encontraron 341 referencias sobre agendas de investigación previas y documentos de estrategias de las cuales se eliminaron 295 por no ser relevantes. Tras revisar el texto completo de los 46 documentos, se 
seleccionaron 24 de ellos (2-25) (figura 1). El tipo del diseño de la mayoría de los estudios encontrados fue de corte transversal $^{4}$. Los estudios y documentos incluidos aportaron informacion sobre las brechas y topicos de investigacion que fueron presentados en el cuestionario inicial.

Partiendo de este proceso, los autores elaboraron la encuesta, que incorporó 11 áreas (políticas públicas, determinantes de la enfermedad renal crónica, etiología, prevención primaria, diagnóstico, tratamiento, prestación de servicios, recursos humanos, información, financiación, determinantes sociales) y 49 tópicos (de los 77 resultantes de la primera ronda).

\section{Características de los participantes}

La encuesta se envió a 83 personas de habla hispana y a 38 de habla inglesa y respondió el $46,2 \%$. Para la segunda ronda, y utilizando el método Delphi adaptado para la priorización de los temas considerados más relevantes en la primera vuelta, la encuesta sólo se envió a los que habían respondido a la primera. En esta segunda vuelta, la encuesta se mandó a 56 participantes en español y a 16 en inglés procedentes, que en conjunto se encontraban en 16 países: Argentina, Brasil, Canadá, Colombia, Costa Rica, Cuba, El Salvador, España, Estados Unidos de América, Francia, Guatemala, Honduras, India, México, Nicaragua y Panamá. El 54\% de ellos pertenecían a algún país de Centroamérica o México.

\section{Prioridades de investigación}

Se seleccionaron finalmente 18 tópicos de investigación, enmarcados en las 10 áreas siguientes (en una de las áreas, los encuestados no consideraron prioritario ninguno de los tópicos): políticas públicas, determinantes de la salud, etiología, diagnóstico y tratamiento de la enfermedad renal crónica, prevención primaria, prestación de servicios, recursos humanos, sistemas de información y financiamiento. Se estimaron los umbrales de decisión y las puntuaciones de cada ítem (la información sobre los umbrales se

\footnotetext{
Los lectores interesados pueden solicitar al autor de correspondencia la lista de los documentos encontrados, así como la de las referencias de los estudios publicados que se seleccionaron en la cual se indica el país donde se realizó el estudio, su diseño, el tipo y el área de investigación y el tema investigado.
}

CUADRO 1. Lista de prioridades de investigación calificadas de alta importancia

\begin{tabular}{|c|c|}
\hline Área & Tópico \\
\hline \multirow[t]{2}{*}{ Políticas públicas } & $\begin{array}{l}\text { Investigación desde la perspectiva de la política pública y el punto de vista } \\
\text { de la salud ocupacional del abordaje de la vigilancia que debe realizarse para } \\
\text { identificar situaciones de riesgo y detectar casos de enfermedad renal } \\
\text { crónica de origen no tradicional (ERCnT) }\end{array}$ \\
\hline & $\begin{array}{l}\text { Investigación sobre medidas efectivas desde el punto de vista de la salud } \\
\text { ocupacional para la prevención y detección temprana de la enfermedad renal } \\
\text { crónica (ERC) de los pacientes expuestos a agroquímicos o sustancias } \\
\text { toxicas por su ocupación }\end{array}$ \\
\hline Determinantes de la salud & $\begin{array}{l}\text { Investigación de determinantes ambientales como posibles factores } \\
\text { etiológicos de la ERCnT }\end{array}$ \\
\hline Etiología & $\begin{array}{l}\text { Investigación de los factores de riesgo de ERCnT (exposiciones a agentes de } \\
\text { riesgo, actividades ocupacionales de riesgo (incluidos el golpe de calor y la } \\
\text { deshidratación crónica), metales pesados, sustancias nefrotóxicas, } \\
\text { intoxicación por agroquímicos) }\end{array}$ \\
\hline \multirow[t]{2}{*}{ Prevención primaria } & $\begin{array}{l}\text { Investigación de las intervenciones dirigidas al lugar de trabajo, al ambiente } \\
\text { o a los individuos en el lugar de trabajo expuestos a factores de riesgo } \\
\text { relacionados con el desarrollo de ERCnT }\end{array}$ \\
\hline & $\begin{array}{l}\text { Investigación sobre la implementación de un sistema de vigilancia de las } \\
\text { exposiciones de riesgo y el manejo inicial del paciente con ERCnT }\end{array}$ \\
\hline \multirow[t]{2}{*}{$\begin{array}{l}\text { Diagnóstico de la enfermedad renal } \\
\text { crónica }\end{array}$} & $\begin{array}{l}\text { Investigación en procesos de investigación para proponer pruebas de } \\
\text { cribado de la ERCnT, como, por ejemplo, biomarcadores de daño temprano }\end{array}$ \\
\hline & $\begin{array}{l}\text { Investigación para el diagnóstico de la ERCnT frente a la ERC convencional } \\
\text { en población laboralmente activa }\end{array}$ \\
\hline \multirow[t]{4}{*}{$\begin{array}{l}\text { Tratamiento de la enfermedad renal } \\
\text { crónica }\end{array}$} & $\begin{array}{l}\text { Investigación sobre la generación de intervenciones clínicas que permitan } \\
\text { detener la progresión de la ERCnT }\end{array}$ \\
\hline & $\begin{array}{l}\text { Evaluación de intervenciones costo-efectivas en el sistema de salud para la } \\
\text { prestación de servicios a pacientes con ERCnT }\end{array}$ \\
\hline & $\begin{array}{l}\text { Investigaciones para el desarrollo de guías de práctica clínica para mejorar } \\
\text { la respuesta de los servicios de salud frente al manejo integral y oportuno } \\
\text { de la ERCnT }\end{array}$ \\
\hline & $\begin{array}{l}\text { Investigacion sobre las estrategias efectivas para la implementación de } \\
\text { guías de práctica clínica para la atención del paciente con ERCnT en } \\
\text { comunidades agrícolas }\end{array}$ \\
\hline \multirow[t]{2}{*}{ Prestación de servicios } & $\begin{array}{l}\text { Investigación de los procesos de prestación de servicios de salud } \\
\text { necesarios para garantizar la atención integral del paciente con ERCnT }\end{array}$ \\
\hline & $\begin{array}{l}\text { Investigación de las necesidades de infraestructura y tecnologías requeridas } \\
\text { para la atención integral del paciente con ERCnT }\end{array}$ \\
\hline \multirow[t]{2}{*}{ Recursos humanos } & $\begin{array}{l}\text { Investigación de las competencias de los profesionales de la salud que } \\
\text { presten sus servicios en la atención de los pacientes con ERCnT }\end{array}$ \\
\hline & $\begin{array}{l}\text { Investigación de la oferta de recursos humanos en salud necesarios para } \\
\text { atender integralmente al paciente con ERCnT }\end{array}$ \\
\hline Sistema de información & Investigación en sistemas de vigilancia epidemiológica de la ERCnT \\
\hline Financiamiento & $\begin{array}{l}\text { Evaluación del impacto presupuestario en el sistema de salud de la } \\
\text { prestación creciente de servicios de salud a pacientes con ERCnT }\end{array}$ \\
\hline
\end{tabular}

puede pedir a los autores, ya que no se ha incluido en este artículo). En el cuadro 1 se presenta la agenda de investigación propuesta y basada en el proceso de priorización. En el anexo 1 se encuentra la lista completa de temas y tópicos priorizados, y en el anexo 2, el análisis de estas prioridades con el método fuzzy sets. Por otro lado, además de los factores etiológicos, sobre todo los ocupacionales y los ambientales, se otorgó gran relevancia a aspectos relacionados con la respuesta de los sistemas de salud, como el abordaje de la prestación de servicios, los recursos humanos, el financiamiento y la atención primaria.

\section{DISCUSIÓN}

Un primer resultado de esta consulta revela que la investigación sobre la ERC$\mathrm{nT}$ es aun relativamente escasa en los países de Centroamérica, sobre todo si se tiene en cuenta la magnitud y complejidad de esta epidemia $(1,2,5,16)$. De hecho, la búsqueda bibliográfíca en noviembre de 2016 sólo identificó 47 estudios primarios publicados en los últimos 5 años, más de dos terceras partes ( 35 de 47) realizados en Nicaragua (17) y El Salvador (18) y el $25 \%$ restante en Costa Rica (4), Guatemala (2) y México (6). Nicaragua y El Salvador son los países que 
mayor evidencia han aportado sobre el tema y esto se puede explicar por el hecho de que sean los países más afectados por la epidemia $(1,2,5,16)$. Sin embargo, también pone de relieve la necesidad de conocer el problema en mayor profundidad, particularmente en otros países de la Región que tienen características geográficas, socioeconómicas, ocupacionales y ambientales similares y por las migraciones de la población trabajadora en Centroamérica (29-32).

El hecho de que el diseño de la mayoría de los estudios fuese de corte transversal limita el establecimiento de los factores de riesgo y el conocimiento de la etiología de la ERCnT en las comunidades agrícolas. En este contexto, sobresale un número creciente de estudios enfocados en el modelo causal del estrés térmico (golpe de calor) y la deshidratación crónica y pocos estudios se han dirigido a identificar de manera rigurosa factores agroquímicos específicos (o sus combinaciones) entre otros factores causales $(16,25,29)$. Cuando estas exposiciones se notifican, se describen de manera general como grupo ("agroquímicos") sin una medición adecuada de las características de la exposición al componente específico. Es también llamativo que pocos grupos de investigación concentran la investigación sobre ERCnT, lo que pone de relieve la necesidad de disponer de una amplia variedad de perfiles de investigadores y grupos de investigación (por ejemplo, sobre ciencias básicas, investigación en políticas y sistemas de salud, cualitativa, epidemiología ambiental y ocupacional) que aporten métodos innovadores y fortalezcan la calidad metodológica de los estudios.

Por otra parte, muy pocos estudios se han enfocado en las necesidades de los sistemas de salud para abordar la ERCnT (33). El amplio abanico de prioridades de investigación identificadas por los expertos, expresado tanto en la variedad de áreas como de tópicos, traduce no sólo las dificultades metodológicas para evaluar los factores causales de la ERCnT a lo largo del tiempo $(2,5,7,8,15)$, sino las dificultades que tienen los sistemas de salud para abordar la prevención, el diagnóstico temprano y el manejo de la enfermedad $(2,5,7,8,15)$. La priorización de aspectos como el conocimiento de los factores etiológicos de la enfermedad y de la incorporación de sistemas de vigilancia desde el punto de vista de la salud ocupacional y ambiental pone de manifiesto la necesidad de incentivar los procesos de investigación epidemiológica y a las instituciones encargadas de la gestión de la salud pública de manera integral (4). La priorización de la investigación en sistemas y servicios de salud, el financiamiento y los recursos humanos para el abordaje de la ERCnT subrayan la necesidad de diseñar, emprender y evaluar acciones sectoriales e intersectoriales para abordar la enfermedad (13, 31-33).

Es esencial prestar atención a estos aspectos mencionados para promover la implementación adecuada de las prioridades de investigación. Por una parte, existen diferencias entre los países, las áreas urbanas y rurales, la afectación predominante de las comunidades agrícolas frecuentemente migrantes y el abordaje según los niveles de acción del sistema de salud $(16,32,33)$. Como se mencionó, la inversión actual y los recursos humanos existentes en investigación para abarcar la complejidad de la ERCnT han sido escasos. Los fondos para las investigaciones frecuentemente provienen de fuentes externas a la Región, lo que, en algunas ocasiones, limita la toma de decisiones a nivel nacional sobre los aspectos que son prioritarios o el enfoque que se da a los problemas. Si se quiere progresar en llenar las brechas de conocimiento, es imperioso cubrir de manera integral todos los aspectos relacionados con la enfermedad y por ello es clave realizar un trabajo mancomunado de los financiadores y los patrocinadores de la investigación.

Asimismo, es importante tener en cuenta que esta agenda de investigación ha de actualizarse periódicamente, dado que las dinámicas del binomio salud-enfermedad, así como los esfuerzos de los sistemas de salud de los países afectados, pueden modificar dichas prioridades. Es aconsejable que este proceso de actualización se adapte a escala nacional según las necesidades de cada país para garantizar el establecimiento de prioridades contextualizadas. La agenda que se propone permite fortalecer la salud pública basada en evidencias como estrategia intersectorial entre la investigación en salud y la política pública a través de la toma de decisiones, construyendo confianza y generando evidencia científica válida para optimizar la toma de decisiones $(26,31)$.

Desde el punto de vista del método planteado en este estudio, el análisis con fuzzy sets se ha aplicado a una serie de sistemas de análisis complejos: optimización de tiempos, optimización de recursos o análisis de prioridades basadas en preferencias. (27). Sin embargo, en el ámbito de la salud se han realizado relativamente pocos estudios con modelos complejos que utilicen estos métodos en procesos de priorización de necesidades en salud. Algunas experiencias previas habían utilizado modelos de colas con disciplina prioritaria o modelos de decisión de colas $(34,35)$. El uso de estos métodos tiene tres ventajas frente a otros de priorización: 1) permiten priorizar teniendo en cuenta la experiencia y el perfil de quien responde, 2) generan un valor umbral y un valor de decisión por dominio y por ítem para poder establecer que realmente es una prioridad, y 3) son métodos no paramétricos de decisión, y por ello permiten trabajar con muestras pequeñas (34). Estos métodos requieren seleccionar verdaderos "expertos" en el área o temática que se desea priorizar, ya que reduce la imprecisión de la estimación de los umbrales de toma de decisión (27). Algunos investigadores han señalado que este modelo de decisión es el que mejor se acerca a la identificación de prioridades reales y a la concepción completa de las necesidades que se quieren enmarcar y solventar (35).

Como todo ejercicio de priorización, el realizado en este estudo tiene limitaciones. A pesar de haber realizado una búsqueda sistemática de estudios publicados y otros documentos sobre prioridades de investigación en ERC, es posible que no se hayan identificado algunos tópicos importantes. La selección de los participantes se basó en identificar a aquellos que hubieran publicado estudios primarios y en sugerencias de expertos de diversas áreas. Sin embargo, es difícil asegurar que todos los puntos de vista se hayan tenido en cuenta. Asimismo, el porcentaje de respuesta en estudios que usan consenso formal e informal tiende a ser bajo. En el presente estudio, para mejorar esta tasa de respuesta, se decidió realizar la encuesta de manera virtual, lo que optimizó el porcentaje de respuesta hasta un $50 \%$. La encuesta se hizo en dos idiomas y se incorporaron participantes de fuera de la Región, porque se tuvo en cuenta que varios investigadores extranjeros habían participado en estudios relacionados con el tema en Centroamérica.

El proceso de priorización utilizado para elaborar la agenda observó las 
pautas de la ética procedimental y buscó poner en práctica el marco de rendición de cuentas para las decisiones razonables con el fin de asegurar que la priorización resultante fuese éticamente aceptable. Por ello, se ha seguido un proceso transparente, explicitado de antemano, enfocado en las consideraciones pertinentes para el tema y respetando la condición de ejecución que es crucial en este marco.

Paa concluir, se espera que este ejercicio para establecer las prioridades regionales de investigación permita generar una serie de acciones conjuntas entre los países de la Región afectados por la ERCnT a través de políticas públicas, estrategias de salud pública efectivas y sistemas de salud que garanticen resolver las necesidades de las poblaciones. La agenda subregional establecida aporta elementos clave para que los países de Centroamérica y el Consejo de Ministros de Salud de Centroamérica (COMISCA) impulsen iniciativas subregionales de salud e investigación para abordar de manera integral la ERCnT reforzando la cooperación y la colaboración mutua. Los sistemas de salud bien organizados, accesibles y resilientes garantizan el abordaje integral y temprano de las comunidades en riesgo de padecer esta enfermedad. La construcción de un modelo causal puede tener un impacto importante en la generación de un plan de acción en salud dirigido a aquellos factores de riesgo modificables que se hayan identificado gracias a la investigación.

\section{REFERENCIAS}

1. García-Trabanino R, Hernández C, Rosa A, Domínguez Alonso J, en nombre del Fondo Social de Emergencia para la Salud (FSES) del cantón Tierra Blanca, departamento de Usulután, El Salvador. Incidence, mortality, and prevalence of end-stage chronic renal disease in the Bajo Lempa region of $\mathrm{El}$ Salvador: A ten-year community registry. Nefrologia. 2016;36(5):517-22.

2. Ordunez $P$, Martinez R, Reveiz L, Chapman E, Saenz C, Soares da Silva A, et al. Chronic kidney disease epidemic in Central America: urgent public health action is needed amid causal uncertainty. PLoS Negl Trop Dis. 2014;8(8):e3019.

3. Orantes $C M$, Herrera $R$, Almaguer $M$, Brizuela EG, Hernández CE, Bayarre $\mathrm{H}$, et al. Chronic kidney disease and associated risk factors in the Bajo Lempa region of El Salvador: Nefrolempa study, 2009. MEDICC Rev. 2011;13(4):14-22.

4. Almaguer M, Herrera R, Orantes CM. Chronic kidney disease of unknown etiology in agricultural communities. MEDICC Rev. 2014;16(2):9-15.

5. Instituto Nacional de Salud. Encuesta nacional de enfermedades crónicas no transmisibles en población adulta en El Salvador, ENECA-ELS (2014-2015). San Salvador: INS; 2015. Disponible en https://www.salud.gob.sv/archivos/comunicaciones/archivos_comunicados 2017 / pdf/presentaciones_evento2003 2017/01-ENECA-ELS-2015.pdf Acceso el 4 de diciembre de 2017.

6. Torres C, Aragón A, González M, López I, Jakobsson K, Elinder CG, et al. Decreased kidney function of unknown cause in Nicaragua: a community-based survey. Am J Kidney Dis. 2010;55(3):485-96.

7. Correa-Rotter R, Wesseling C, Johnson RJ. CKD of Unknown Origin in Central America: The Case for a Mesoamerican Nephropathy. Am J Kidney Dis. 2014; 63(3):506-20.
8. Cusumano AM, González Bedat MC. Chronic kidney disease in Latin America: time to improve screening and detection. Clin J Am Soc Nephrol. 2008;3(2):594-600.

9. Lozier M, Turcios-Ruiz RM, Noonan G, Ordunez P. Chronic kidney disease of nontraditional etiology in Central America: a provisional epidemiologic case definition for surveillance and epidemiologic studies. Rev Panam Salud Publica. 2016;(40)5: 294-300.

10. Ferreiro A, Álvarez-Estévez G, CerdasCalderón M, Cruz-Trujillo Z, Mena E, Reyes M, et al. Confirmed clinical case of chronic kidney disease of nontraditional causes in agricultural communities in Central America: a case definition for surveillance. Rev Panam Salud Publica. 2016; 40(5)301-08.

11. Escamilla-Cejudo JA, Lara Báez J, Peña R, Ruiz Luna PL, Ordunez P. Optimización del registro de muerte por enfermedad renal crónica en las comunidades agrícolas de América Central. Rev Panam Salud Publica. 2016;40(5):285-93.

12. Pan American Health Organization. Epidemic of Chronic Kidney Disease in Agricultural Communities in Central America. Case definitions, methodological basis and approaches for public health surveillance. Washington, DC: PAHO; 2017.

13. Jha V, Garcia-Garcia G, Iseki K, Li Z, Naicker S, Plattner B, et al. Chronic kidney disease: global dimension and perspectives. Lancet 2013;382(9888):260-72.

14. Jayatilake N, Mendis S, Maheepala P, Mehta FR, CKDu National Research Project Team. Chronic kidney disease of uncertain aetiology: prevalence and causative factors in a developing country. BMC Nephrol. 2013;14:180.

15. Glaser J, Lemery J, Rajagopalan B, Diaz HF, Garcia-Trabanino R, Taduri G, et al. Climate change and the emergent
Agradecimientos. Los autores agradecen especialmente las aportaciones de todos los participantes en el establecimiento de prioridades.

Financiación. Esta publicación ha sido realizada con el apoyo financiero de la Agencia Española de Cooperación Internacional para el Desarrollo (AECID).

Conflictos de interés. Ninguno declarado por los autores.

Declaración. Las opiniones expresadas en este manuscrito son responsabilidad de los autores y no reflejan necesariamente los criterios ni la política de la RPSP/PAJPH ni de la OPS o la AECID. epidemic of CKD from heat stress in rural communities: The case for heat stress neprhopathy. Clin J Am Soc Nephrol. 2016; 11(8):1472-83.

16. Silva LC, Ordunez P. Chronic kidney disease in agricultural Central American communities: challenges for epidemiology and Public Health. MEDICC Review. 2014;16(2):66-71.

17. Ordunez P, Saenz C, Martinez R, Chapman E, Reveiz L, Becerra F. The epidemic of chronic kidney disease in Central America. Lancet Glob Health. 2014;2(8):e440-1.

18. Pan American Health Organization (2014) Resolution CD52.R1. Chronic kidney disease in agricultural communities in Central America. Washington, DC: PAHO 2013. Disponible en: http://www.paho. org/hq/index.php?option=com_content\& view $=$ article $\&$ id $=8833 \&$ Itemid $=40033 \& 1-$ ang=en Acceso el 21 de octubre de 2016.

19. Lunyera J, Mohottige D, Von Isenburg M, Jeuland M, Patel UD, Stanifer JW. CKD of Uncertain Etiology: A Systematic Review. Clin J Am Soc Nephrol. 2016;11(3):379-85.

20. Wesseling C, Crowe J, Hogstedt C, Jakobsson K, Lucas R5, Wegman DH. First International Research Workshop on the Mesoamerican Nephropathy. Resolving the enigma of the mesoamerican nephropathy: a research workshop summary. Am J Kidney Dis. 2014;63(3):396-404.

21. Jayasumana C, Orantes C, Herrera R, Almaguer M, Lopez L, Silva LC, et al. Chronic interstitial nephritis in agricultural communities: a worldwide epidemic with social, occupational and environmental determinants. Nephrol Dial Transplant. 2016;32(2):234-41.

22. Weaver VM, Fadrowski JJ, Jaar BG. Global dimensions of chronic kidney disease of unknown etiology (CKDu): a modern era environmental and/or occupational nephropathy? BMC Nephrology. 2015; 16:145. 
23. López-Marín L, Chávez Y, García XA, Flores WM, García YM, Herrera R, et al. Histopathology of chronic kidney disease of unknown etiology in Salvadoran agricultural communities. MEDICC Rev. 2014; 16(2):49-54.

24. Orantes-Navarro CM, Herrera-Valdés R, Almaguer-López M, López-Marín L, VelaParada XF, Hernandez-Cuchillas M, et al. Toward a Comprehensive Hypothesis of Chronic Interstitial Nephritis in Agricultural Communities. Adv Chronic Kidney Dis. 2017;24(2):101-6.

25. Ramírez O, McClean MD, Amador JJ, Brooks D. An epidemic of chronic kidney disease in Central America: an overview. J Epidemiol Comm Health. 2013;67(1):1-3.

26. Reveiz L, Chapman E, Flórez CE, Torres R. Priorities for health policy and systems research focused on human resources in health. Rev Panam Salud Publica. 2013; 34(5):295-303.

27. Verma AK, Srividya A, Prabhu Gaonkar R. Fuzzy set solutions for optimal maintenance strategy selection. OPSEARCH J. 2007; 44(3):261-76.

28. Smithson M. Fuzzy set inclusion linking fuzzy set methods with main-stream techniques. Sociol Meth Res. 2005;33(4): 431-61.

29. Ramírez-Rubio O, McClean MD, Amador JJ, Brooks DR. An epidemic of chronic kidney disease in Central America: an overview. Postgrad Med J. 2013;89(1049):123-5.

30. Loría Bolaños $R$, Partanen $T$, Berrocal $M$, Alvárez B, Córdoba L. Determinants of health in seasonal migrants: coffee harvesters in Los Santos, Costa Rica. Int J Occup Environ Health. 2008;14(2):129-37.

31. Martin-Cleary C, Ortiz A. CKD hotspots around the world: where, why and what the lessons are. A CKJ review series. Clin Kidney J. 2014;7(6):519-23.

32. Levin A, Tonelli M, Bonventre J, Coresh J, Donner J, Fogo A, et al. Global kidney health 2017 and beyond: a roadmap for closing gaps in care, research, and policy. Lancet. 2017;390(10105):1888-917.
33. Lobb R, Colditz GA. Implementation Science and its application to population health. Annu Rev Public Health. 2013;34: 235-51.

34. Kahraman C, Bozdag C, Ruan D, FahriOzok A. Fuzzy sets approaches to statistical parametric and nonparametric tests. International J Intelligent Syst. 2004 19(11):1069-87.

35. Ishikawa A, Amagasa $M$, Shiga $T$, Tomizawa G, Tatsuta R, Mieno H. The max-min Delphi method and fuzzy Delphi method via fuzzy integration. Fuzzy Sets Systems. 1993;55(3):241-53.

Manuscrito recibido el 11 de enero de 2017. Aceptado para publicación, tras revisión, el 7 de noviembre de 2017.

Introduction. In 2013, the PAHO Member States recognized the epidemic of chronic kidney disease of non-traditional causes (CKDnT) as a serious public health problem. This article describes the establishment of research priorities to comprehensively address CKDnT in Central America.

Establishing research Methods. Following a search of the literature for research studies carried out in Central America and prior research agendas on CKD, a virtual survey was conducted using the Delphi methodology. The respondents were identified from various sources. The first round sought to refine and add research topics and to prioritize those deemed most relevant. The second round prioritized the most relevant topics. A fuzzy-sets analysis was carried out to estimate decision thresholds and scores for each topic.

Results. The survey was sent to 83 Spanish-speaking and 38 English-speaking prospective respondents. The response rate was $46.2 \%$. For the second round, the survey was sent to 56 Spanish-speaking and 16 English-speaking first-round respondents. Eighteen topics within 10 research areas were prioritized: public policies, determinants, etiology, diagnosis and treatment of CKD, primary prevention, service delivery, human resources, information systems, and funding. Research on CKDnT was found to be scarce and restricted to certain topics.

Conclusions. In addition to etiological factors, great importance was assigned to aspects related to the health system response, including service delivery approaches, human resources, funding, and occupational and environmental aspects.

Keywords Chronic renal insufficiency; research; health priorities; health systems; occupational health services. 
RESUMO

Determinação de prioridades em pesquisa da doença renal crônica associada a causas não tradicionais na América Central

Palavras-chave
Introdução. Em 2013, os Estados Membros da OPAS reconheceram a epidemia de doença renal crônica associada a causas não tradicionais como um sério problema de saúde pública. Este artigo descreve a determinação de prioridades em pesquisa para uma abordagem ampla da doença renal crônica associada a causas não tradicionais na América Central.

Métodos. Foi estruturada uma pesquisa virtual com o uso da metodologia Delphi e foi feita uma busca dos estudos realizados na América Central e das agendas de pesquisa anteriores sobre doença renal crônica. Os entrevistados eram provenientes de fontes diversas. Na primeira rodada, buscou-se refinar e acrescentar tópicos de pesquisa e priorizar os mais relevantes. Na segunda rodada, foram priorizados os tópicos mais relevantes. Foi realizada uma análise com o uso de conjuntos nebulosos para estimar limiares de decisão e pontuações por tópico.

Resultados. A pesquisa foi enviada primeiramente a 83 indivíduos falantes da língua espanhola e 38 falantes da língua inglesa, com taxa de resposta de $46,2 \%$. $\mathrm{Na}$ segunda rodada, a pesquisa foi enviada aos 56 falantes da língua espanhola e 16 falantes da língua inglesa que haviam respondido a primeira rodada da pesquisa. Foram priorizados 18 tópicos de pesquisa distribuídos em 10 áreas: políticas públicas, determinantes, etiologia, diagnóstico e tratamento da doença renal crônica, prevenção primária, prestação de serviços, recursos humanos, sistemas de informação e financiamento. Foi verificado que a pesquisa em doença renal crônica associada a causas não tradicionais é escassa e está restrita a determinados tópicos.

Conclusões. Além dos fatores etiológicos, foi dada grande relevância a aspectos relacionados à resposta dos sistemas de saúde, incluindo o método de prestação de serviços, recursos humanos, financiamento e aspectos ocupacionais e ambientais.

Insuficiência Renal Crônica; Pesquisa; Prioridades em Saúde; Sistemas de Saúde; Serviços de Saúde do Trabalhador 